\title{
A novel benzothiazole synthesis by cyclization of ketenimines bearing sulfenylimine fragments. Unexpected sulfur to carbon migration of an imino group
}

\author{
Mateo Alajarín, ${ }^{a}{ }^{a}$ Ángel Vidal, ${ }^{a}$ Fulgencio Tovar, ${ }^{a}$ Peter G. Jones, ${ }^{b}$ and Delia Bautista ${ }^{c}$ \\ ${ }^{a}$ Departamento de Química Orgánica, Facultad de Química, Universidad de Murcia, Campus \\ de Espinardo, 30100, Murcia, Spain \\ ${ }^{b}$ Institut für Anorganische und Analytische Chemie, Technische Universität \\ Braunschweig, Postfach 3329, 38023 Braunschweig, Germany \\ ${ }^{c}$ Servicio Universitario de Instrumentación Científica, Universidad de Murcia, Campus de \\ Espinardo, 30100, Murcia, Spain \\ E-mail: alajarin@um.es;
}

\section{Dedicated to Professor José Elguero on the occasion of his $\mathbf{7 0}^{\text {th }}$ birthday, and to Professor Pedro Molina on his $60^{\text {th }}$ birthday}

(received 26 Nov 04; accepted 13 Jan 05; published on the web 25 Jan 05)

\begin{abstract}
Transient ketenimines containing sulfenylimine fragments in which both functionalities are linked by an ortho-phenylene scaffold through their nitrogen and sulfur atoms respectively, underwent intramolecular cyclization yielding 2-(iminomethyl)benzothiazoles. These processes involve the formation of a new $\mathrm{C}-\mathrm{S}$ bond and the concomitant migration of the imino group from the sulfur atom of the sulfenylimine fragment to the terminal carbon atom of the ketenimine function.
\end{abstract}

Keywords: Sulfenylimine, ketenimine, cyclization, imino, migration, benzothiazole

\section{Introduction}

$\mathrm{N}$-Sulfenylimines ( $\mathrm{N}$-alkylidenesulfenamides, sulfenimines) are important organic compounds, structurally characterized by a divalent sulfur atom binding an imine function by means of the nitrogen atom $\left[\mathrm{R}^{1}-\mathrm{S}-\mathrm{N}=\mathrm{CR}^{2} \mathrm{R}^{3}\right]$. The $N$-sulfenylimine functional group is the key constituent of some biologically active compounds. Smyth has recently demonstrated that penicillins and cephalosporins bearing an $S$-aminosulfenylimine side chain at the 6- and 7-positions respectively, are prototypical examples of novel classes of $\beta$-lactamase-dependent prodrugs, wherein enzyme-catalyzed cleavage of the $\beta$-lactam ring triggers a rapid expulsion of the $S$ amino moiety. ${ }^{1} \mathrm{~N}$-Sulfenylimines can be prepared by a variety of synthetic methods ${ }^{2}$ that involve 
the use as starting materials of various types of sulfur-containing compounds such as sulfenamides, ${ }^{3}$ disulfides, ${ }^{4}$ sulfenyl halides, ${ }^{5}$ thioketenes, ${ }^{6}$ thioamides, ${ }^{7}$ and other less common substrates. $^{8} \mathrm{~N}$-Sulfenylimines are compounds with very rich chemistry which can be explained on the following grounds: a) the oxidation of the divalent sulfur atom; ${ }^{9}$ b) the nucleophilic character of the nitrogen and sulfur atoms, thus allowing reactions with electrophilic species; ${ }^{10} \mathrm{c}$ ) the electrophilic nature of the iminyl carbon, which is responsible for the addition of a variety of nucleophiles to that carbon; ${ }^{\mathrm{e}, 11}$ and d) the reaction of $N$-sulfenylimines as enolate equivalents with electrophiles. ${ }^{3 \mathrm{a}, 12}$ However, to the best of our knowledge, there are no reports describing the participation of sulfenylimines in cycloaddition reactions, with the sole exception of the cycloaddition of the hexafluoroisopropylideneamidosulfenyl system $\left(\mathrm{CF}_{3}\right)_{2} \mathrm{C}=\mathrm{N}-\mathrm{S}-\mathrm{F}$ with the $\mathrm{CF}_{3} \mathrm{NO}$ diradical. ${ }^{13}$

Over the last few years, we have studied several modes of intramolecular cyclization of imino-ketenimines in which the two reactive functionalities are connected through their nitrogen atoms by different tethers. We have found that such intramolecular [2+2] cycloadditions take place efficiently when the imine and ketenimine functions are supported on an ortho-benzylic scaffold, as in the imino-ketenimines $\mathbf{1}^{14}$ (Scheme 1). The same selective mode of cyclization was found with imino-ketenimines $3{ }^{15}$ in which the $\mathrm{N}=\mathrm{C}$ double bond involved in the cycloaddition is part of an $\mathrm{N}$-acylimino group (Scheme 1).
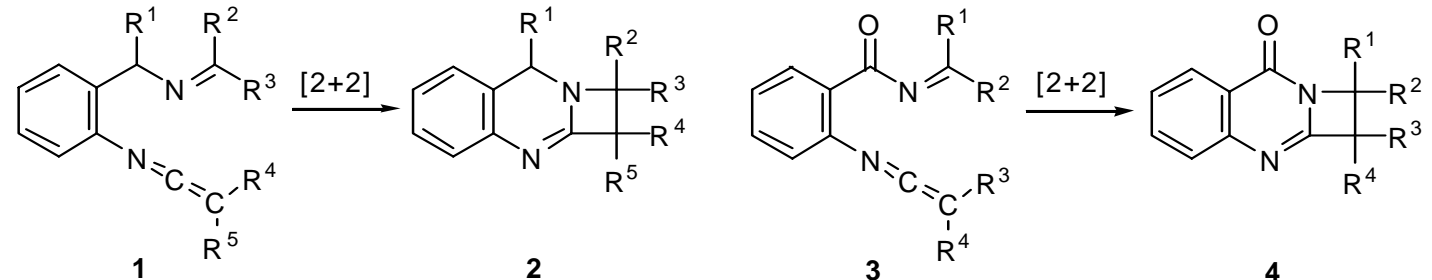

Scheme 1. Intramolecular [2+2] cycloaddition of imino(acylimino)ketenimines on an orthobenzylic scaffold.

Based on these results, we decided to explore whether an $N$-sulfenylimine function could participate in analogous [2+2] cycloadditions involving its iminic $\mathrm{N}=\mathrm{C}$ double bond. To this end we selected as substrates sulfenylimino-ketenimines of general structure $\mathbf{I}$ (structural analogs of compounds 1 and $\mathbf{3}$ in which the benzylic $s p^{3}$ or $s p^{2}$ carbon atom has been replaced by a sulfur atom), which, by intramolecular [2+2] cycloaddition, could provide new heterocyclic compounds II, bearing an azetidine ring fused to a benzothiadiazine system (Scheme 2).

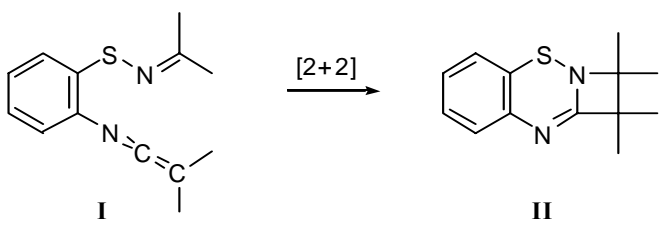

Scheme 2. Planned intramolecular [2+2] cycloaddition of sulfenylimino-ketenimines. 
Herein we describe the results obtained in attempts to prepare some sulfenyliminoketenimines of structure I. These compounds proved to be transient intermediates in cyclization processes that take place by nucleophilic addition of the sulfur atom of the sulfenylimine function into the $s p$ - hybridized carbon atom of the ketenimine fragment, instead of through the planned intramolecular [2+2] cycloaddition of the sulfenylimine with the ketenimine. The products so obtained are 2-substituted benzothiazoles.

\section{Results and Discussion}

The readily available bis-(2-azidophenyl) disulfide 5, in solution in methanol, was treated sequentially with silver nitrate and diphenylketimine or bis-(4-methylphenyl)ketimine - this last step in the presence of triethylamine - to provide the sulfenylimines $\mathbf{6 a}-\mathbf{b}$, which are disubstituted at the iminic carbon atom (Scheme 3). A different synthetic method was necessary for preparing the sulfenylimines $\mathbf{6 c - e}$, monosubstituted at the imine carbon atom. For the preparation of compounds $\mathbf{6 c}-\mathbf{e}$ a solution of bis-(2-azidophenyl) disulfide $\mathbf{5}$ in methanol was treated first with silver nitrate, and then gaseous ammonia was bubbled through the resulting suspension to produce 2-azidobenzenesulfenamide 7 which, in the same reaction flask, reacts with aromatic aldehydes to yield the sulfenylimines $\mathbf{6 c - e}$ (Scheme 3).

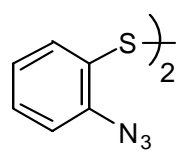

a) $\mathrm{AgNO}_{3}, \mathrm{MeOH}$, reflux, $1 \mathrm{~h}$

b) $\mathrm{HN}=\overbrace{\mathrm{R}^{2}}^{\mathrm{R}^{1}}, \mathrm{Et}_{3} \mathrm{~N}, \mathrm{MeOH}$, r.t., $20 \mathrm{~h}$<smiles>[R]C([R])=NSc1ccccc1N</smiles>

$6 a-b$

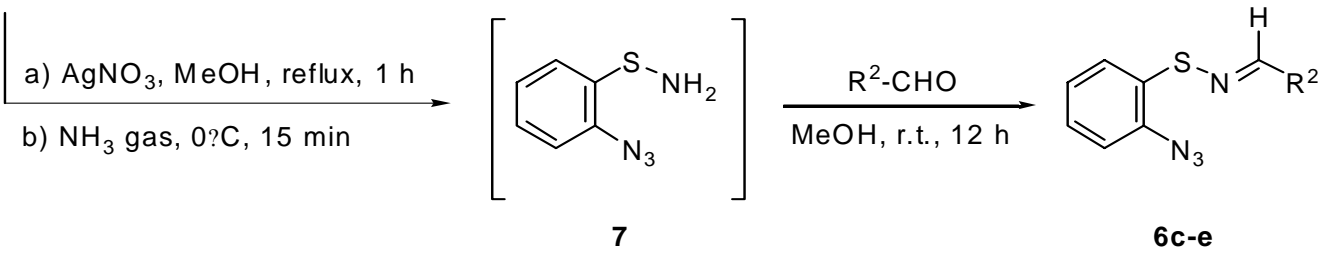

Scheme 3. Methods for the preparation of the 2-azidobenzenesulfenamides 6.

6a: $\mathrm{R}^{1}=\mathrm{R}^{2}=\mathrm{C}_{6} \mathrm{H}_{5} ; \mathbf{6} \mathbf{b}: \mathrm{R}^{1}=\mathrm{R}^{2}=4-\mathrm{CH}_{3}-\mathrm{C}_{6} \mathrm{H}_{4} ; \mathbf{6 c}: \mathrm{R}^{1}=\mathrm{H}, \mathrm{R}^{2}=4-\mathrm{Br}_{-} \mathrm{C}_{6} \mathrm{H}_{4}$;

6d: $\mathrm{R}^{1}=\mathrm{H}, \mathrm{R}^{2}=4-\mathrm{Cl}-\mathrm{C}_{6} \mathrm{H}_{4} ; \mathbf{6 e}: \mathrm{R}^{1}=\mathrm{H} ; \mathrm{R}^{2}=3,5-\left(\mathrm{CH}_{3} \mathrm{O}\right)_{2}-\mathrm{C}_{6} \mathrm{H}_{3}$

Staudinger reactions ${ }^{16}$ of the 2-azidobenzenesulfenamides 6 with trimethylphosphine, in toluene solution at room temperature, gave the trimethylphosphazenes $\mathbf{8}$, which were used in the following step without isolation, because of the hydrolytic sensitivity of the phosphazene group. The conversion $\mathbf{6} \rightarrow \mathbf{8}$ could be followed by IR and ${ }^{31} \mathrm{P}$ - NMR. Thirty minutes after the addition of trimethylphosphine to the toluene solution of the azide $\mathbf{6}$, the IR spectra of the reaction 
mixtures showed two strong absorptions at $1438 \mathrm{~cm}^{-1}$ and $1121 \mathrm{~cm}^{-1}$ corresponding to the phosphazene grouping, and the band corresponding to the azide group (near $2100 \mathrm{~cm}^{-1}$ ) of the starting material did not appear. The ${ }^{31} \mathrm{P}-\mathrm{NMR}$ spectra of compounds $\mathbf{8}$, obtained by removing the solvent from a small fraction of the reaction mixture, showed one signal around $10 \mathrm{ppm}$ attributable to the $N$-aryltrimethylphosphazene grouping. The toluene solutions of compounds 8 were subsequently treated at room temperature with disubstituted ketenes (diphenylketene and methylphenylketene). In less than one hour, IR analyses of the reaction mixtures showed no cumulenic absorptions in the $1900-2100 \mathrm{~cm}^{-1}$ range, and after chromatographic purification the 2-substituted benzothiazoles 10 were isolated in moderate to good yields (Scheme 4, Table 1). ${ }^{17}$ The $E / Z$ configuration of the $\mathrm{N}=\mathrm{C}$ bond in compounds $10 \mathrm{~d}-\mathrm{g}$ (in which $\mathrm{R}^{1} \neq \mathrm{R}^{2}$ ) has not been elucidated, although they were apparently obtained as single diastereoisomers.

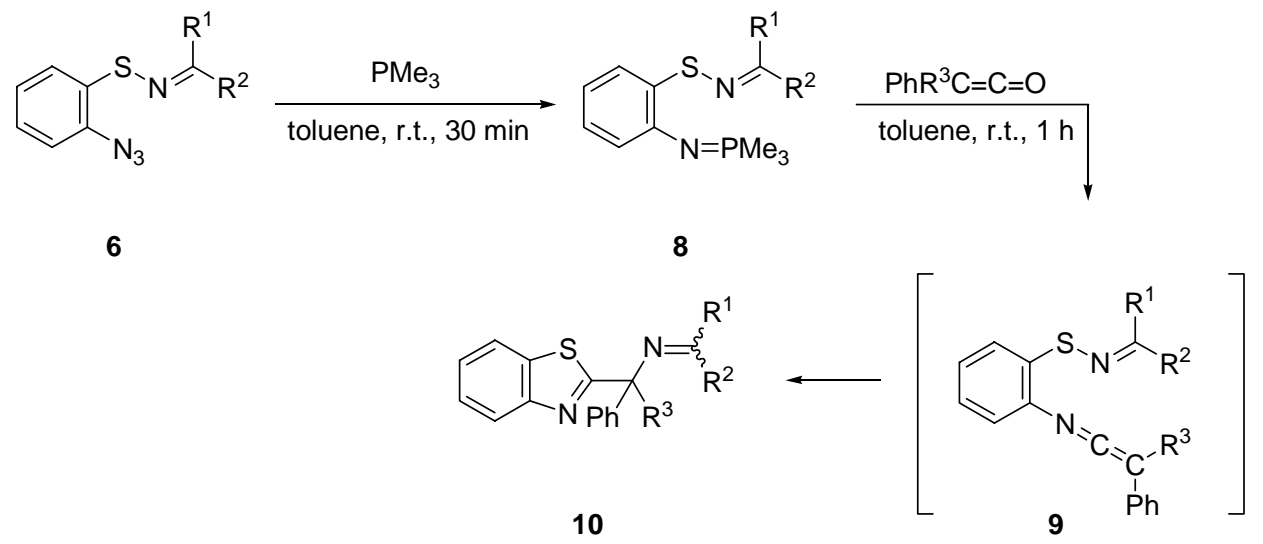

Scheme 4. Synthetic sequence leading to the benzothiazoles $\mathbf{1 0 .}$

Table 1. 2-Substituted benzothiazoles 10

\begin{tabular}{lllll}
\hline Compound & $\mathrm{R}^{1}$ & $\mathrm{R}^{2}$ & $\mathrm{R}^{3}$ & Yield (\%) \\
\hline $\mathbf{1 0 a}$ & $\mathrm{C}_{6} \mathrm{H}_{5}$ & $\mathrm{C}_{6} \mathrm{H}_{5}$ & $\mathrm{C}_{6} \mathrm{H}_{5}$ & 88 \\
$\mathbf{1 0 b}$ & $\mathrm{C}_{6} \mathrm{H}_{5}$ & $\mathrm{C}_{6} \mathrm{H}_{5}$ & $\mathrm{CH}_{3}$ & 56 \\
$\mathbf{1 0 c}$ & $4-\mathrm{CH}_{3}-\mathrm{C}_{6} \mathrm{H}_{4}$ & $4-\mathrm{CH}_{3}-\mathrm{C}_{6} \mathrm{H}_{4}$ & $\mathrm{C}_{6} \mathrm{H}_{5}$ & 63 \\
$\mathbf{1 0 d}$ & $\mathrm{H}$ & $4-\mathrm{Br}_{-} \mathrm{C}_{6} \mathrm{H}_{4}$ & $\mathrm{C}_{6} \mathrm{H}_{5}$ & 64 \\
$\mathbf{1 0}$ & $\mathrm{H}$ & $4-\mathrm{Br}_{-} \mathrm{C}_{6} \mathrm{H}_{4}$ & $\mathrm{CH}_{3}$ & 41 \\
$\mathbf{1 0 f}$ & $\mathrm{H}$ & $4-\mathrm{Cl}-\mathrm{C}_{6} \mathrm{H}_{4}$ & $\mathrm{C}_{6} \mathrm{H}_{5}$ & 89 \\
$\mathbf{1 0 g}$ & $\mathrm{H}$ & $3,5-\left(\mathrm{CH}_{3} \mathrm{O}\right)_{2}-\mathrm{C}_{6} \mathrm{H}_{3}$ & $\mathrm{C}_{6} \mathrm{H}_{5}$ & 50 \\
\hline
\end{tabular}

The structural elucidation of the benzothiazoles $\mathbf{1 0}$ was achieved from their analytical and spectral data, and was eventually confirmed by an X-ray structure determination of $\mathbf{1 0 a}\left(\mathrm{R}^{1}=\mathrm{R}^{2}\right.$ $=\mathrm{R}^{3}=\mathrm{C}_{6} \mathrm{H}_{5}$ ) (Figure 1). ${ }^{18}$ The IR spectra of the benzothiazoles $\mathbf{1 0}$ show strong absorptions in the region 1627-1652 $\mathrm{cm}^{-1}$, attributable to the imine function on the side chain. In the ${ }^{1} \mathrm{H}-\mathrm{NMR}$ spectra of compounds $\mathbf{1 0 d}, \mathbf{f}, \mathbf{g}\left(\mathrm{R}^{1}=\mathrm{H} ; \mathrm{R}^{3}=\mathrm{C}_{6} \mathrm{H}_{5}\right)$ the proton at the iminic carbon resonates at $\delta$ $=7.65-7.71$, whereas for $10 \mathrm{e}\left(\mathrm{R}^{1}=\mathrm{H} ; \mathrm{R}^{3}=\mathrm{CH}_{3}\right)$ the same proton is observed at $\delta=8.13$. 


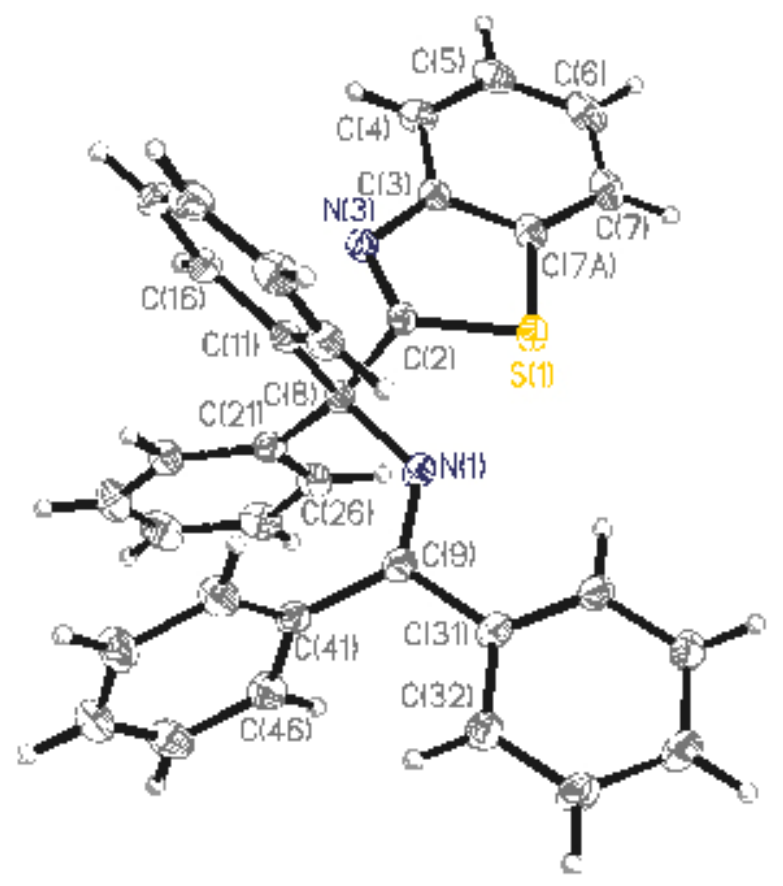

Figure 1. Thermal ellipsoid plot (50\% probability level) for 10a. Selected bond lengths $(\AA)$ and angles (deg.): $\mathrm{S}(1)-\mathrm{C}(7 \mathrm{~A})=1.7372$ (11), $\mathrm{S}(1)-\mathrm{C}(2)=1.7519(10), \mathrm{N}(1)-\mathrm{C}(9)=1.2825$ (13), $\mathrm{N}(1)-$ $\mathrm{C}(8)=1.4743(12), \mathrm{C}(2)-\mathrm{N}(3)=1.2907(13), \mathrm{N}(3)-\mathrm{C}(3)=1.3933 \quad(13), \mathrm{C}(7 \mathrm{~A})-\mathrm{S}(1)-\mathrm{C}(2)=88.44$ (5), $\mathrm{C}(9)-\mathrm{N}(1)-\mathrm{C}(8)=124.47 \quad(9), \mathrm{N}(3)-\mathrm{C}(2)-\mathrm{C}(8)=124.04 \quad(9), \mathrm{N}(3)-\mathrm{C}(2)-\mathrm{S}(1)=116.70$ (8), $\mathrm{C}(8)-\mathrm{C}(2)-\mathrm{S}(1)=119.20(7), \mathrm{C}(2)-\mathrm{N}(3)-\mathrm{C}(3)=110.42$ (9), $\mathrm{N}(3)-\mathrm{C}(3)-\mathrm{C}(4)=125.17$ (10), $\mathrm{N}(3)-$ $\mathrm{C}(3)-\mathrm{C}(7 \mathrm{~A})=114.74(9), \mathrm{C}(7)-\mathrm{C}(7 \mathrm{~A})-\mathrm{S}(1)=129.23$ (9), $\mathrm{C}(3)-\mathrm{C}(7 \mathrm{~A})-\mathrm{S}(1)=109.68(8), \mathrm{N}(1)-$ $\mathrm{C}(8)-\mathrm{C}(11)=110.56(8), \mathrm{N}(1)-\mathrm{C}(8)-\mathrm{C}(2)=103.90(8), \mathrm{N}(1)-\mathrm{C}(8)-\mathrm{C}(21)=112.46(8), \mathrm{N}(1)-\mathrm{C}(9)-$ $\mathrm{C}(31)=115.87(9), \mathrm{N}(1)-\mathrm{C}(9)-\mathrm{C}(41)=127.34(9)$.

In the ${ }^{13} \mathrm{C}-\mathrm{NMR}$ spectra of the benzothiazoles $\mathbf{1 0}$ the aliphatic quaternary carbon atom linked to $\mathrm{C} 2$ resonates at $\delta=68.1-78.2 \mathrm{ppm}$. For compounds $\mathbf{1 0 d}-\mathbf{g}\left(\mathrm{R}^{1}=\mathrm{H}\right)$ the signal of the methine carbon of the imine function appeared at $\delta=159.2-161.8$.

In the crystal structure of $\mathbf{1 0 a}$, the benzothiazole ring is planar, with a mean deviation of only $0.01 \AA$. The $\mathrm{S}-\mathrm{C}$ and the $\mathrm{N}-\mathrm{C}$ bonds of the benzothiazole ring are in the normal range.

A reasonable mechanism for explaining the conversion $\mathbf{8} \rightarrow \mathbf{1 0}$ is shown in Scheme 5: an azaWittig $^{19}$ reaction between the trimethylphosphazenes $\mathbf{8}$ and the ketenes should give the transient sulfenylimino-ketenimines $\mathbf{9}$, which probably undergo cyclization to the zwitterionic intermediate 11 by nucleophilic addition of the sulfur atom of the sulfenylimine function onto the electrophilic central carbon atom of the ketenimine moiety. Next, the intramolecular 1,3migration of the imino group from the sulfur atom of the zwitterionic intermediate $\mathbf{1 1}$ to the carbon atom linked to carbon $\mathrm{C} 2$ of the benzothiazole ring should lead to compounds $\mathbf{1 0}$. Thus, the formation of the benzothiazoles $\mathbf{1 0}$ from the sulfenylimino-ketenimines $\mathbf{9}$ involves an electrophilic intramolecular migration of the imino group ${ }^{20}$ from the sulfur atom of the sulfenylimine function to the terminal carbon atom of the ketenimine fragment. 
The benzothiazole nucleus is of particular interest, especially in the field of medicinal chemistry, because many useful therapeutic agents contain this heterocyclic system. For example, benzothiazoles bearing a 2-(4-aminophenyl) substituent at the 2-position represent a novel class of potent and selective antitumor agents. ${ }^{21}$ Benzothiazoles are commonly synthesized by sequential or simultaneous formation of one S-C and one N-C bond starting from 2aminoarenethiols. $^{22}$

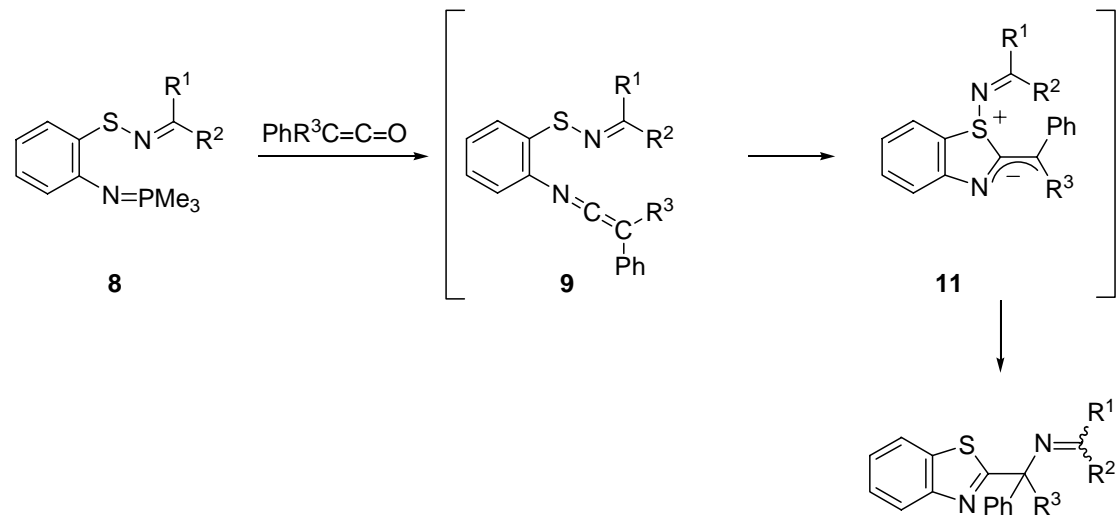

10

Scheme 5. Proposed mechanism for the conversion $\mathbf{8} \rightarrow \mathbf{1 0}$.

\section{Experimental Section}

General Procedures. All melting points were determined on a Kofler hot-plate melting point apparatus and are uncorrected. IR spectra were recorded as films or Nujol emulsions on a Nicolet Impact-400 spectrophotometer. NMR spectra were recorded on a Bruker AC-200 or a Varian Unity-300. Chemical shifts ( $\delta$ in ppm) are given from internal $\mathrm{SiMe}_{4}(0.0 \mathrm{ppm})$ for ${ }^{1} \mathrm{H}-\mathrm{NMR}$ and $\mathrm{CDCl}_{3}(77.1 \mathrm{ppm})$ for ${ }^{13} \mathrm{C}-\mathrm{NMR}$. Mass spectra were recorded on a Hewlett-Packard 5993 instrument. Microanalyses were performed on a Carlo Erba EA-1108 instrument.

The crystal structure of $\mathbf{1 0 a}$ was determined by single crystal X-ray diffraction. Measurements were recorded using a Bruker Smart 1000 CCD diffractometer with monochromated Mo- $K \alpha$ radiation in the $\omega$-scan mode. The structure was solved by direct methods and refined anisotropically on $F^{2}$ (program system SHELXL-97, G.M. Sheldrick, University of Göttingen, Germany). Hydrogen atoms were included using a riding model.

Materials. Bis-(2-Azidophenyl) disulfide $\mathbf{5}^{23}$ diphenylketimine, ${ }^{24}$ bis-(4-methylphenyl)ketimine $^{24}$ diphenylketene ${ }^{25}$ and methylphenylketene ${ }^{26}$ were prepared following experimental procedures previously reported.

General procedure for the preparation of the 2 -azidobenzenesulfenamides $6 \mathbf{a}-\mathbf{b}$

A suspension of bis-(2-azidophenyl) disulfide $5(0.3 \mathrm{~g}, 1 \mathrm{mmol})$ and silver nitrate $(0.17 \mathrm{~g}$, $1.01 \mathrm{mmol})$ in methanol $(5 \mathrm{ml})$ was heated at reflux for 1 hour. After cooling at room 
temperature, a solution of the corresponding ketimine $(0.75 \mathrm{mmol})$ and triethylamine $(0.079 \mathrm{~g}$, $0.078 \mathrm{mmol})$ in the same solvent $(2 \mathrm{ml})$ was added. The resulting heterogeneous mixture was stirred at room temperature for 20 hours. Then dichloromethane $(20 \mathrm{ml})$ was added, and the solids were separated by filtration and washed with more dichloromethane $(2 \times 10 \mathrm{ml})$. The combined organic extracts were washed with water $(2 \times 20 \mathrm{ml})$ and dried over anhydrous magnesium sulfate. After filtration, the solvent was removed under reduced pressure and the resulting material was chromatographed on a silica gel column using hexanes/diethyl ether [9:1, (v/v)] as eluent.

$\mathbf{N}$-Diphenylmethylene-2-azidobenzenesulfenamide (6a). Yield $45 \%$. $\mathrm{Mp} 127-128^{\circ} \mathrm{C}\left(\mathrm{Et}_{2} \mathrm{O}\right.$, colorless prisms); IR (Nujol) 2131 (s), 2091 (s), 1572 (w), 1444 (s), 1304 (m), 1287 (m), 1056 (w), $756(\mathrm{~s}), 695(\mathrm{~m}) \mathrm{cm}^{-1}$; ${ }^{1} \mathrm{H}-\mathrm{NMR}\left(\mathrm{CDCl}_{3}\right) \delta: 7.00-7.05(\mathrm{~m}, 1 \mathrm{H}), 7.11-7.20(\mathrm{~m}, 3 \mathrm{H}), 7.27-$ $7.31(\mathrm{~m}, 4 \mathrm{H}), 7.43-7.46(\mathrm{~m}, 3 \mathrm{H}), 7.55-7.59(\mathrm{~m}, 2 \mathrm{H}), 8.01-8.06(\mathrm{~m}, 1 \mathrm{H}) ;{ }^{13} \mathrm{C}-\mathrm{NMR}\left(\mathrm{CDCl}_{3}\right) \delta$ : $118.1,125.6,125.8,126.5,127.5,128.0,128.3,129.1,129.5,130.0,134.6(\mathrm{~s}), 137.6$ (s), 137.7 (s), 139.0 (s), 165.3 (s). Anal. Calcd for $\mathrm{C}_{19} \mathrm{H}_{14} \mathrm{~N}_{4} \mathrm{~S}$ (330.09): C, 69.07; H, 4.27; N, 16.96. Found: C, 69.21; H, 4.13; N, 16.87\%.

$\mathbf{N - B i s - ( 4 - m e t h y l p h e n y l ) m e t h y l e n e - 2 - a z i d o b e n z e n e s u l f e n a m i d e ~ ( 6 b ) . ~ Y i e l d ~ 4 6 \% . ~ M p ~ 1 3 5 - ~}$ $136^{\circ} \mathrm{C}$ (Et ${ }_{2} \mathrm{O}$, colorless prisms); IR (Nujol) 2134 (vs), 2098 (vs), 1613 (w), 1576 (m), 1506 (m), 1306 (s), 1292 (s), 1158 (w), 1056 (m), 1036 (w), 824 (m), 752 (s), 718 (m) cm ${ }^{-1}$; ${ }^{1} \mathrm{H}-\mathrm{NMR}$ $\left(\mathrm{CDCl}_{3}\right) \delta: 2.36(\mathrm{~s}, 3 \mathrm{H}), 2.44(\mathrm{~s}, 3 \mathrm{H}), 7.09(\mathrm{dd}, 1 \mathrm{H}, J=1.1,7.7 \mathrm{~Hz}), 7.15(\mathrm{~d}, 2 \mathrm{H}, J=8.1 \mathrm{~Hz})$, 7.20 (td, $1 \mathrm{H}, J=1.4,7.7 \mathrm{~Hz}), 7.23-7.27$ (m, $3 \mathrm{H}), 7.32$ (d, $2 \mathrm{H}, J=8.1 \mathrm{~Hz}), 7.54$ (d, $2 \mathrm{H}, J=$ $8.1 \mathrm{~Hz}), 8.11(\mathrm{dd}, 1 \mathrm{H}, J=1.4,7.7 \mathrm{~Hz}) ;{ }^{13} \mathrm{C}-\mathrm{NMR}\left(\mathrm{CDCl}_{3}\right) \delta: 21.4,21.6,118.0,125.6,125.7$, 126.3, 127.4, 128.1, 129.0, 129.6, 131.6 (s), 134.4 (s), 134.9 (s), 136.6 (s), 139.4 (s), 140.2 (s), 165.6 (s); EI-MS m/z: $358\left(\mathrm{M}^{+}, 11\right), 179$ (100). Anal. Calcd for $\mathrm{C}_{21} \mathrm{H}_{18} \mathrm{~N}_{4} \mathrm{~S}$ (358.46): C, 70.36; H, 5.06; N, 15.63. Found: C, 70.09; H, 5.13; N, 15.55\%.

\section{General procedure for the preparation of the 2-azidobenzenesulfenamides $6 \mathrm{c}-\mathrm{e}$}

A suspension of bis-(2-azidophenyl) disulfide $5(0.3 \mathrm{~g}, 1 \mathrm{mmol})$ and silver nitrate $(0.17 \mathrm{~g}$, $1.01 \mathrm{mmol})$ in methanol $(5 \mathrm{ml})$ was refluxed for 1 hour. After cooling the reaction mixture at $0^{\circ} \mathrm{C}$ in an ice bath, ammonia was bubbled through the solution for nearly 15 minutes. The aldehyde $(1.2 \mathrm{mmol})$ was added and the heterogeneous reaction mixture was stirred at room temperature for 12 hours. Then dichloromethane $(20 \mathrm{ml})$ was added, and the solids were separated by filtration and washed with more dichloromethane $(2 \times 10 \mathrm{ml})$. The combined organic phase was washed with water $(2 \times 20 \mathrm{ml})$ and dried over anhydrous magnesium sulfate. After filtration, the solvent was removed under reduced pressure and the resulting material was chromatographed on a silica gel column using hexanes/diethyl ether $[9: 1,(\mathrm{v} / \mathrm{v})]$ as eluent.

$\mathrm{N}$-(4-Bromobenzylidene)-2-azidobenzenesulfenamide (6c). Yield 44\%. Mp $112-114^{\circ} \mathrm{C}\left(\mathrm{Et}_{2} \mathrm{O}\right.$, colorless prisms); IR (Nujol) v: 2131 (vs), 2095 (vs), 1577 (s), 1293 (vs), 1064 (m), 1012 (m), 959 (w), 928 (w), 819 (m), 746 (s), $719(\mathrm{~m}) \mathrm{cm}^{-1} ;{ }^{1} \mathrm{H}-\mathrm{NMR}\left(\mathrm{CDCl}_{3}\right)$ 8: 7.19 (dd, $1 \mathrm{H}, J=1.3$, $7.8 \mathrm{~Hz}), 7.25(\mathrm{td}, 1 \mathrm{H}, J=1.3,7.8 \mathrm{~Hz}), 7.32(\mathrm{td}, 1 \mathrm{H}, J=1.6,7.8 \mathrm{~Hz}), 7.52-7.58(\mathrm{~m}, 4 \mathrm{H}), 7.84$ $(\mathrm{dd}, 1 \mathrm{H}, J=1.6,7.8 \mathrm{~Hz}), 8.40(\mathrm{~s}, 1 \mathrm{H}) ;{ }^{13} \mathrm{C}-\mathrm{NMR}\left(\mathrm{CDCl}_{3}\right) \delta: 118.4,125.0$ (s), 125.7, 128.1, 
128.2, 128.3 (s), 128.8, 132.0, 135.1 (s), 136.3 (s), 156.5; EI-MS m/z: $334\left(\mathrm{M}^{+}+2,6\right), 332\left(\mathrm{M}^{+}\right.$, 6), 197 (100). Anal. Calcd for $\mathrm{C}_{13} \mathrm{H}_{9} \mathrm{BrN}_{4} \mathrm{~S}$ (333.21): C, 46.86; H, 2.72; N, 16.81. Found: C, 46.59; H, 2.66; N, 16.99\%.

$\mathrm{N}$-(4-Chlorobenzylidene)-2-azidobenzenesulfenamide (6d). Yield 38\%. $\mathrm{Mp} 132^{\circ} \mathrm{C}\left(\mathrm{Et}_{2} \mathrm{O}\right.$, colorless prisms); IR (Nujol) v: 2129 (vs), 2045 (vs), 1595 (w), 1577 (w), 1488 (m), 1441 (s), 1293 (s), 1124 (w), 1085 (w), 958 (w), 822 (m), 745 (s) cm ${ }^{-1} ;{ }^{1} \mathrm{H}-\mathrm{NMR}\left(\mathrm{CDCl}_{3}\right)$ 8: 7.09-7.25 (m, $3 \mathrm{H}), 7.30(\mathrm{~d}, 2 \mathrm{H}, J=8.5 \mathrm{~Hz}), 7.56(\mathrm{~d}, 2 \mathrm{H}, J=8.5 \mathrm{~Hz}), 7.78(\mathrm{dd}, 1 \mathrm{H}, J=1.8,7.3 \mathrm{~Hz}), 8.35$ (s, $1 \mathrm{H}) ;{ }^{13} \mathrm{C}-\mathrm{NMR}\left(\mathrm{CDCl}_{3}\right) \delta: 118.3,125.7,127.9,128.1,128.4$ (s), 128.6, 129.1, 134.7 (s), 136.2 (s), 136.5 (s), 156.4. Anal. Calcd for $\mathrm{C}_{13} \mathrm{H}_{9} \mathrm{ClN}_{4} \mathrm{~S}$ (288.02): C, 54.07; H, 3.14; N, 19.40. Found: C, 53.93; H, 3.27; N, 19.55\%.

$\mathrm{N}$-(3,5-Dimethoxybenzylidene)-2-azidobenzenesulfenamide (6e). Yield 55\%. Mp 74-76 ${ }^{\circ} \mathrm{C}$ $\left(\mathrm{CH}_{2} \mathrm{Cl}_{2} / \mathrm{Et}_{2} \mathrm{O}\right.$, colorless prisms); IR (Nujol) v: 2135 (vs), 2097 (vs), 1602 (vs), 1566 (s), 1296 (vs), 1208 (s), 1153 (vs), 1059 (s), 832 (m), 748 (s) cm ${ }^{-1} ;{ }^{1} \mathrm{H}-\mathrm{NMR}\left(\mathrm{CDCl}_{3}\right) \delta: 3.83$ (s, $\left.6 \mathrm{H}\right), 6.52$ $(\mathrm{t}, 1 \mathrm{H}, J=2.3 \mathrm{~Hz}), 6.85(\mathrm{~d}, 2 \mathrm{H}, J=2.3 \mathrm{~Hz}), 7.19(\mathrm{dd}, 1 \mathrm{H}, J=1.2,7.7 \mathrm{~Hz}), 7.23(\mathrm{td}, 1 \mathrm{H}, J=$ $1.2,7.7 \mathrm{~Hz}), 7.32(\mathrm{td}, 1 \mathrm{H}, J=1.5,7.7 \mathrm{~Hz}), 7.84(\mathrm{dd}, 1 \mathrm{H}, J=1.5,7.7 \mathrm{~Hz}), 8.37(\mathrm{~s}, 1 \mathrm{H}) ;{ }^{13} \mathrm{C}-$ NMR $\left(\mathrm{CDCl}_{3}\right) \delta:$ 55.6, 103.1, 105.4, 118.3, 125.7, 128.0, 128.3, 136.4 (s), 138.1 (s), 157.8, 161.0 (s); EI-MS m/z: $314\left(\mathrm{M}^{+}, 14\right), 122$ (100). Anal. Calcd for $\mathrm{C}_{15} \mathrm{H}_{14} \mathrm{~N}_{4} \mathrm{O}_{2} \mathrm{~S}$ (314.37): C, 57.31; H, 4.49; N, 17.82. Found: C, 57.09; H, 4.62; N, 17.77\%.

General procedure for the preparation of the 2-(iminomethyl)benzothiazoles 10 Trimethylphosphine ( $1.5 \mathrm{mmol}, 1.5 \mathrm{ml}$ of a $1 \mathrm{M}$ toluene solution) was added to a solution of the corresponding 2-azidobenzenesulfenamide $6(1.5 \mathrm{mmol})$ in dry toluene $(10 \mathrm{ml})$, and the reaction mixture was stirred at room temperature until the evolution of nitrogen ceased (15-30 minutes). Then diphenylketene or methylphenylketene $(1.5 \mathrm{mmol})$ was added, and the mixture was allowed to stir at room temperature for 1 hour. The solvent was removed under reduced pressure and the crude material purified by column chromatography.

2-[1,1-Diphenyl-1-( $\mathrm{N}$-diphenylmethylideneamino)]methylbenzothiazole(10a). Chromatography on silica gel with hexanes/diethyl ether [9:1, (v/v)]. Yield $88 \%$. Mp $237^{\circ} \mathrm{C}\left(\mathrm{CH}_{2} \mathrm{Cl}_{2} / \mathrm{Et}_{2} \mathrm{O}\right.$, colorless prisms); IR (Nujol) v: 1627 (s), 1598 (m), 1507 (m), 1493 (m), 1312 (m), 1279 (m),

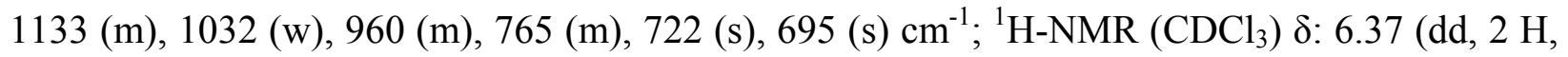
$J=1.0,7.5 \mathrm{~Hz}), 6.75(\mathrm{t}, 2 \mathrm{H}, J=7.8 \mathrm{~Hz}), 6.90-7.03(\mathrm{~m}, 7 \mathrm{H}), 7.14-7.28(\mathrm{~m}, 7 \mathrm{H}), 7.30-7.38(\mathrm{~m}$, $3 \mathrm{H}), 7.74-7.82(\mathrm{~m}, 3 \mathrm{H}) ;{ }^{13} \mathrm{C}-\mathrm{NMR}\left(\mathrm{CDCl}_{3}\right) \delta: 76.3(\mathrm{~s}), 123.4,124.4,125.4,126.8,126.9$, 127.0, 127.4, 127.5, 128.2, 129.2, 129.7, 130.7, 135.9 (s), 137.9 (s), 141.2 (s), 143.5 (s), 154.7 (s), 169.6 (s), 184.7 (s); EI-MS m/z: $480\left(\mathrm{M}^{+}, 33\right), 165$ (100). Anal. Calcd for $\mathrm{C}_{33} \mathrm{H}_{24} \mathrm{~N}_{2} \mathrm{~S}$ (480.62): C, 82.47; H, 5.03; N, 5.83. Found: C, 82.31; H, 5.24; N, 5.97\%.

Crystal data for 10a: $\mathrm{C}_{33} \mathrm{H}_{24} \mathrm{~N}_{2} \mathrm{~S}, M=480.60$, Monoclinic, space group $P 2{ }_{1} / c, a=11.6241(11), b$ $=12.5986(11), c=16.8391(15) \AA, \beta=96.770$ (3), $V=2448.8(4) \AA^{3}, Z=4, \lambda$ (Mo $\left.K \alpha\right)=$ $0.71073 \AA, T=133 \mathrm{~K}, \mu=0.16 \mathrm{~mm}^{-1}, 27844$ reflections measured, 7157 unique $\left(R_{\text {int }}=0.0256\right)$ used in all calculations. The final $R 1$ was $0.037[I>2 \sigma(I)]$ with $w R 20.105$ (all data). 
2-[1-( $N$-Diphenylmethylideneamino)-1-methyl-1-phenyl]methylbenzothiazole (10b). Chromatography on silica gel with hexanes/diethyl ether [9:1, (v/v)]. Yield 56\%. Mp $187-188^{\circ} \mathrm{C}$ (Et ${ }_{2} \mathrm{O}$, colorless prisms); IR (Nujol) v: 1633 (vs), 1597 (m), 1579 (m), 1510 (m), 1458 (vs), 1448 (vs), 1313 (m), 1128 (m), 1026 (s), 952 (w), 760 (s), 728 (s), 703 (vs) cm ${ }^{-1}$; ${ }^{1} \mathrm{H}-\mathrm{NMR}\left(\mathrm{CDCl}_{3}\right) \delta$ : $1.93(\mathrm{~s}, 3 \mathrm{H}), 6.58(\mathrm{~d}, 2 \mathrm{H}, J=6.9 \mathrm{~Hz}), 7.07-7.13(\mathrm{~m}, 5 \mathrm{H}), 7.15-7.28(\mathrm{~m}, 3 \mathrm{H}), 7.31-7.45(\mathrm{~m}, 5$ $\mathrm{H}), 7.76-7.81(\mathrm{~m}, 2 \mathrm{H}), 7.85-7.89(\mathrm{~m}, 1 \mathrm{H}), 7.93-7.98(\mathrm{~m}, 1 \mathrm{H}) ;{ }^{13} \mathrm{C}-\mathrm{NMR}\left(\mathrm{CDCl}_{3}\right) \delta: 25.8,68.1$ (s) $121.7,123.0,124.3,125.5,126.8,127.0,127.5,127.6,127.8,128.2,128.7,130.5,136.0$ (s), 137.7 (s), 140.9 (s), 146.3 (s), 154.7 (s), 168.3 (s), 185.7 (s); EI-MS m/z: 418 (M+, 20), 238 (100). Anal. Calcd for $\mathrm{C}_{28} \mathrm{H}_{22} \mathrm{~N}_{2} \mathrm{~S}$ (418.15): C, 80.35; H, 5.30; N, 6.69. Found: C, 80.47; H, 5.51; $\mathrm{N}, 6.47 \%$.

2-\{1-[N-Bis-(4-methylphenyl)methylideneamino]-1,1-diphenyl $\}$ methylbenzothiazole (10c). Chromatography on silica gel with hexanes/ethyl acetate $[9: 1,(\mathrm{v} / \mathrm{v})]$. Yield $63 \%$. Mp $201^{\circ} \mathrm{C}$ $\left(\mathrm{Et}_{2} \mathrm{O}\right.$, colorless prisms); ${ }^{1} \mathrm{H}-\mathrm{NMR}\left(\mathrm{CDCl}_{3}\right) \delta: 2.19$ (s, $\left.3 \mathrm{H}\right), 2.39$ (s, $\left.3 \mathrm{H}\right), 6.32$ (d, $2 \mathrm{H}, J=7.9$ $\mathrm{Hz}), 6.62$ (d, $2 \mathrm{H}, J=7.9 \mathrm{~Hz}), 7.03-7.08$ (m, $6 \mathrm{H}), 7.21$ (d, $2 \mathrm{H}, J=7.9 \mathrm{~Hz}), 7.27-7.36$ (m, $6 \mathrm{H})$, $7.76(\mathrm{~d}, 2 \mathrm{H}, J=7.9 \mathrm{~Hz}), 7.87(\mathrm{~d}, 2 \mathrm{H}, J=7.9 \mathrm{~Hz}) ;{ }^{13} \mathrm{C}-\mathrm{NMR}\left(\mathrm{CDCl}_{3}\right) \delta: 21.2,21.5,76.1(\mathrm{~s})$, $121.5,123.3,124.3,125.3,126.7,127.3,127.9,128.9,129.1,129.7,135.2(\mathrm{~s}), 135.9$ (s), 136.5 (s), 138.7 (s), 141.0 (s), 143.7 (s), 154.7 (s), 169.7 (s), 185.1 (s); EI-MS m/z: 508 (M+ 13 ), 300 (100). Anal. Calcd for $\mathrm{C}_{35} \mathrm{H}_{28} \mathrm{~N}_{2} \mathrm{~S}$ (508.68): C, 82.64; H, 5.55; N, 5.51. Found: C, 82.45; H, 5.34; $\mathrm{N}, 5.87 \%$.

2-\{1-[N-(4-Bromobenzylidene)amino]-1,1-diphenyl\} methylbenzothiazole (10d). Chromatography on silica gel with hexanes/diethyl ether [9:1, (v/v)]. Yield $=64 \%$. Mp 205-206 ${ }^{\circ} \mathrm{C}\left(\mathrm{Et}_{2} \mathrm{O}\right.$, colorless prisms); IR (Nujol) v: 1645 (vs), 1593 (s), 1132 (m), 1068 (m), 1012 (s), 896 (m), 831 (m), 754 (s), 725 (s), 704 (vs) cm ${ }^{-1}$; ${ }^{1} \mathrm{H}-\mathrm{NMR}\left(\mathrm{CDCl}_{3}\right) \delta: 7.28-7.39(\mathrm{~m}, 12 \mathrm{H}), 7.59$ (d, $2 \mathrm{H}, J=$ $8.6 \mathrm{~Hz}), 7.71(\mathrm{~s}, 1 \mathrm{H}), 7.76(\mathrm{~d}, 2 \mathrm{H}, J=8.6 \mathrm{~Hz}), 7.83-7.88(\mathrm{~m}, 1 \mathrm{H}), 8.00-8.06(\mathrm{~m}, 1 \mathrm{H}) ;{ }^{13} \mathrm{C}-$ NMR $\left(\mathrm{CDCl}_{3}\right) \delta: 78.2$ (s), 121.5, 123.5, 124.8, 125.7, 126.1 (s), 127.8, 128.2, 129.4, 130.1, 132.1, 134.7 (s), 135.6 (s), 142.7 (s), 153.8 (s), 160.7, 179.3 (s); EI-MS m/z: $484\left(\mathrm{M}^{+}+2,5\right), 482$ $\left(\mathrm{M}^{+}, 6\right), 301$ (100). Anal. Calcd for $\mathrm{C}_{27} \mathrm{H}_{19} \mathrm{BrN}_{2} \mathrm{~S}$ (483.43): C, 67.08; H, 3.96; N, 5.79. Found: C, $66.91 ; \mathrm{H}, 3.77$; N, 5.87\%.

2-\{1-[N-(4-Bromobenzylidene)amino]-1-methyl-1-phenyl $\}$ methylbenzothiazole (10e). Chromatography on silica gel with hexanes/diethyl ether [9:1, (v/v)]. Yield 41\%. IR (film) v: 1649 (vs), 1591 (s), 1513 (vs), 1490 (vs), 1439 (s), 1071 (vs), 1013 (vs), 824 (s), 763 (vs) cm ${ }^{-1}$;

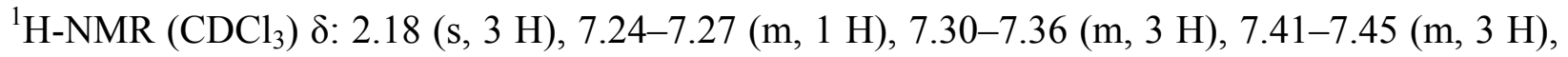
$7.57(\mathrm{~d}, 2 \mathrm{H}, J=8.4 \mathrm{~Hz}), 7.73(\mathrm{~d}, 2 \mathrm{H}, J=8.4 \mathrm{~Hz}), 7.85-7.87(\mathrm{~m}, 1 \mathrm{H}), 8.00(\mathrm{~d}, 1 \mathrm{H}, J=7.9 \mathrm{~Hz})$, $8.13(\mathrm{~s}, 1 \mathrm{H}) ;{ }^{13} \mathrm{C}-\mathrm{NMR}\left(\mathrm{CDCl}_{3}\right) \delta: 27.3,70.2$ (s), 121.7, 123.2, 124.7, 125.7, 125.8 (s), 127.2, 127.6, 128.5, 130.0, 132.0, 135.1 (s), 135.8 (s), 144.5 (s), 153.7 (s), 159.2, 180.2 (s); EI-MS m/z: $422\left(\mathrm{M}^{+}+2,4\right), 420\left(\mathrm{M}^{+}, 5\right), 239$ (100). Anal. Calcd for $\mathrm{C}_{22} \mathrm{H}_{17} \mathrm{BrN}_{2} \mathrm{~S}$ (421.36): C, 62.71; $\mathrm{H}$, 4.07 ; N, 6.65. Found: C, 62.58; H, 3.89; N, 6.54\%.

2-\{1-[N-(4-Chlorobenzylidene)amino]-1,1-diphenyl\}methylbenzothiazole (10f). Chromatography on silica gel with hexanes/diethyl ether [9:1, (v/v)]. Yield 89\%. IR (Nujol) v: 1648 (s), 1598 (m), 1495 (m), 1447 (s), 1298 (m), 1088 (m), 1012 (m), 898 (m), 755 (s), 702 (s) cm ${ }^{-1}$; ${ }^{1} \mathrm{H}-\mathrm{NMR}$ 
$\left(\mathrm{CDCl}_{3}\right) \delta: 7.21-7.29(\mathrm{~m}, 11 \mathrm{H}), 7.32-7.38(\mathrm{~m}, 4 \mathrm{H}), 7.65(\mathrm{~s}, 1 \mathrm{H}), 7.74-7.77(\mathrm{~m}, 3 \mathrm{H}), 7.95(\mathrm{~d}, 1$ $\mathrm{H}, J=8.1 \mathrm{~Hz}) ;{ }^{13} \mathrm{C}-\mathrm{NMR}\left(\mathrm{CDCl}_{3}\right) \delta: 78.2(\mathrm{~s}), 121.5,123.5,124.8,125.7,127.8,128.2,129.1$, 130.1, 134.4 (s), 135.6 (s), 137.6 (s), 138.2 (s), 142.7 (s), 153.9 (s), 160.7, 179.3 (s); EI-MS m/z: $440\left(\mathrm{M}^{+}+2,5\right), 438\left(\mathrm{M}^{+}, 15\right), 120$ (100). Anal. Calcd for $\mathrm{C}_{27} \mathrm{H}_{19} \mathrm{ClN}_{2} \mathrm{~S}$ (438.10): C, 73.87; $\mathrm{H}$, $4.36 ; \mathrm{N}, 6.38$. Found: C, 74.01; H, 4.39; N, 6.16\%.

2-\{1-[N-(3,5-Dimethoxybenzylidene)amino]-1,1-diphenyl $\}$ methylbenzothiazole (10g). Chromatography on silica gel with hexanes/ethyl acetate [9:1, (v/v)]. Yield 50\%. IR (Nujol) v: 1652 (s), 1599 (vs), 1507 (m), 1303 (s), 1268 (m), 1208 (vs), 1161 (vs), 1066 (s), 847 (m), 739 (vs), 703 (s) cm ${ }^{-1} ;{ }^{1} \mathrm{H}-\mathrm{NMR}\left(\mathrm{CDCl}_{3}\right) \delta: 3.79$ (s, $\left.3 \mathrm{H}\right), 3.86(\mathrm{~s}, 3 \mathrm{H}), 6.59$ (t, $\left.1 \mathrm{H}, J=2.3 \mathrm{~Hz}\right), 7.06$ $(\mathrm{d}, 2 \mathrm{H}, J=2.3 \mathrm{~Hz}), 7.29-7.37(\mathrm{~m}, 11 \mathrm{H}), 7.45$ (td, $1 \mathrm{H}, J=1.1,7.9 \mathrm{~Hz}), 7.69$ (s, $1 \mathrm{H}), 7.87$ (d, 1 $\mathrm{H}, J=7.9 \mathrm{~Hz}), 8.03(\mathrm{~d}, 1 \mathrm{H}, J=7.9 \mathrm{~Hz}) ;{ }^{13} \mathrm{C}-\mathrm{NMR}\left(\mathrm{CDCl}_{3}\right) \delta: 55.7,78.1(\mathrm{~s}), 104.0,106.6$, $121.5,123.5,124.7,125.6,127.8,128.2,129.5,135.7$ (s), 137.9 (s), 142.8 (s), 153.9 (s), 161.1 (s), 161.8, 179.7 (s); EI-MS m/z: $464\left(\mathrm{M}^{+}, 9\right), 300$ (100). Anal. Calcd for $\mathrm{C}_{29} \mathrm{H}_{24} \mathrm{~N}_{2} \mathrm{O}_{2} \mathrm{~S}$ (464.59): C, 74.97; H, 5.21; N, 6.03. Found: C, 74.66; H, 5.05; N, 6.26\%.

\section{Acknowledgments}

This work was supported by the MCYT and FEDER (Project BQU2001-0010) and Fundación Séneca-CARM (Project PI-00749/FS/01).

\section{References}

1. (a) Smyth, T. P.; O’Donnell, M. E.; O'Connor, M. J.; Ledger, J. O. St. J. Org. Chem. 1998, 63, 7600. (b) Smyth, T. P.; O'Connor, M. J.; O’Donnell, M. E. J. Org. Chem. 1999, 64, 3132. (c) Smyth, T. P.; O’Donnell, M. E.; O'Connor, M. J.; Ledger, J. O. St. Tetrahedron 2000, 56, 5699.

2. For reviews on the synthesis of N-sulfenylimines, see: (a) Robertson, G. M. Comprehensive Organic Functional Group Transformation, Katritzky, A. R.; Meth-Cohn, O.; Rees, C. W. Eds; Elsevier: Oxford, 1995; Vol. 3, pp 425-441 and 733-856. (b) Claus, K. Chemistry of Sulphenic Acids and their Derivatives, Patai, S. Ed.; Wiley: Chichester, 1990.

3. For examples of preparations of sulfenylimines from sulfenamides, see: (a) Davis, F. A.; Mancinelli, P. A. J. Org. Chem. 1978, 43, 1797. (b) Morimoto, T.; Nezu, Y.; Achiwa, K.; Sekiya, M. J. Chem. Soc., Chem. Commun. 1985, 1584. (c) Refvik, M. D.; Schwan, A. L. Tetrahedron 1996, 52, 8387.

4. For examples of preparations of sulfenylimines from disulfides, see: (a) Davis, F. A.; Slegeir, W. A. R.; Kaminski, J. M. J. Chem. Soc., Chem. Commun. 1972, 634. (b) Davis, F. A.; Slegeir, W. A. R.; Evans, S.; Schwartz, A.; Goff, D. L.; Palmer, R. J. Org. Chem. 1973, 38, 2809. (c) Barton, D. H. R.; Magnus, P. D.; Pennanen, S. I. J. Chem. Soc., Chem. Commun. 
1974, 1007. (d) Brenner, D. G.; Cavolowsky, K. M.; Shepard, K. L. J. Heterocycl. Chem. 1985, 22, 805.

5. For examples of preparations of sulfenylimines from sulfenyl halides, see: (a) Davis, F. A.; Kluger, E. W. J. Am. Chem. Soc. 1976, 98, 302. (b) Ried, W.; Dietschmann, H.; Erle, H. E. Synthesis 1980, 619.

6. For examples of preparations of sulfenylimines from thioketenes, see: Schaumann, E.; Bolte, O.; Behr, H. J. Chem. Soc., Perkin Trans. 1 1990, 182.

7. For examples of preparations of sulfenylimines from thioamides, see: (a) Beddoes, R. L.; Cernik, R. J.; Mills, O. S.; Stansfield, F. J. Chem. Soc., Chem. Commun. 1983, 390. (b) Stansfield, F. J. Chem. Soc., Perkin Trans. 1 1984, 2933.

8. (a) Dj-Forudian, H.; Hudson, R. F.; Record, K. A. F. J. Chem. Soc., Chem. Commun. 1976, 503. (b) Gordon, E. M.; Pluscec, J. J. Org. Chem. 1979, 44, 1218. (c) Pike, S.; Walton, D. R. M. Tetrahedron Lett. 1980, 21, 4989. (d) Rezessy, B.; Toldy, L.; Tóth, G. Tetrahedron Lett. 1992, 33, 6523.

9. (a) Davis, F. A.; Friedman, A. J.; Kluger, E. W. J. Am. Chem. Soc. 1974, 96, 5000. (b) Davis, F. A.; Friedman, A. J.; Nadir, U. K. J. Am. Chem. Soc. 1978, 100, 2844. (c) Davis, F. A.; Chen, B.-C. Chem. Rev. 1992, 92, 919. (d) Davis, F. A.; Reddy, R. T.; Reddy, R. E. J. Org. Chem. 1992, 57, 6387. (e) Yang, T.-K.; Chen, R.-Y.; Lee, D.-S.; Peng, W.-S.; Jiang, Y.-Z.; Mi, A-Q.; Jong, T.-T. J. Org. Chem. 1994, 59, 914. (f) Davis, F. A.; Reddy, R. E.; Szewczyk, J. M.; Reddy, G. V.; Portonovo, P. S.; Zhang, H.; Fanelli, D.; Reddy, R. T.; Zhou, P.; Carroll, P. J. J. Org. Chem. 1997, 62, 2555.

10. (a) Davis, F. A.; Skibo, E. B. J. Org. Chem. 1974, 39, 807. (b) Reck, R.; Jochims, J. C. Chem. Ber. 1982, 115, 1494.

11. (a) Davis, F. A.; Mancinelli, P. A. J. Org. Chem. 1977, 42, 398. (b) Fronza, G.; Fuganti, C.; Grasselli, P.; Pedrochi-Fantoni, G. Tetrahedron Lett. 1981, 22, 5073. (c) Burnett, D. A.; Hart, D. J.; Liu, J. J. Org. Chem. 1986, 51, 1929. (d) Barnes, D. M.; McLaughlin, M. A.; Oie, T.; Rasmussen, M. W.; Stewart, K. D.; Wittenberger, S. J. Org. Lett. 2002, 4, 1427. (e) DeGoey, D. A.; Chen, H.-J.; Flosi, W. J.; Grampovnik, D. J.; Yeung, C. M.; Klein, L. L.; Kempf, D. J. J. Org. Chem. 2002, 67, 5445. (f) Barnes, D. M.; Bhagavatula, L.; DeMattei, J.; Gupta, A.; Hill, D. R.; Manna, S.; McLaughlin, M. A.; Nichols, P.; Premchandran, R.; Rasmussen, M. W.; Tian, Z.; Wittenberger, S. J. Tetrahedron: Asymmetry 2003, 14, 3541.

12. (a) Davis, F. A.; Mancinelli, P. A. J. Org. Chem. 1980, 45, 2597. (b) Branchaud, B. P. J. Org. Chem. 1983, 48, 3531.

13. Varwig, J.; Mews, R. J. Chem. Research (S) 1977, 245.

14. (a) Alajarín, M.; Molina, P.; Vidal, A. Tetrahedron Lett. 1996, 37, 8945. (b) Alajarín, M.; Molina, P.; Vidal, A.; Tovar, F. Tetrahedron 1997, 53, 13449. (c) Alajarín, M.; Vidal, A.; Tovar, F.; Arrieta, A.; Lecea, B.; Cossío, F. P. Chem. Eur. J. 1999, 5, 1106. (d) Cossío, F. P.; Arrieta, A.; Lecea, B.; Alajarín, M.; Vidal, A.; Tovar, F. J. Org. Chem. 2000, 65, 3633. (e) Alajarín, M.; Vidal, A.; Tovar, F.; Ramírez de Arellano, M. C.; Cossío, F. P.; Arrieta, A.; 
Lecea, B. J. Org. Chem. 2000, 65, 7512. (f) Alajarín, M.; Vidal, A.; Tovar, F.; Ramírez de Arellano, M. C. Tetrahedron: Asymmetry 2004, 15, 489.

15. Alajarín, M.; Sánchez-Andrada, P.; Vidal, A.; Tovar, F. J. Org. Chem. in press.

16. (a) Staudinger, H.; Meyer, J. Helv. Chim. Acta 1919, 2, 635. (b) Gololobov, Y. G.; Zhmurova, I. N.; Kasukhin, L. F. Tetrahedron 1981, 37, 437. (c) Gololobov, Y. G.; Kasukhin, L. F. Tetrahedron 1992, 48, 1353.

17. In the reaction of the trimethylphosphazenes $\mathbf{8 c}-\mathbf{e}$ (in which $\mathrm{R}^{1}=\mathrm{H}$ ) with diphenylketene or methylphenylketene small amounts $(9-12 \%)$ of 2-(diphenylmethyl)benzothiazole 13a or 2(1-phenylethyl)benzothiazole 13b were also isolated, along with the corresponding benzonitrile, $\mathrm{R}^{2}-\mathrm{CN}$. The benzothiazoles $\mathbf{1 3}$ should form either by partial decomposition of the benzothiazoles $\mathbf{1 0 d}-\mathbf{g}$,

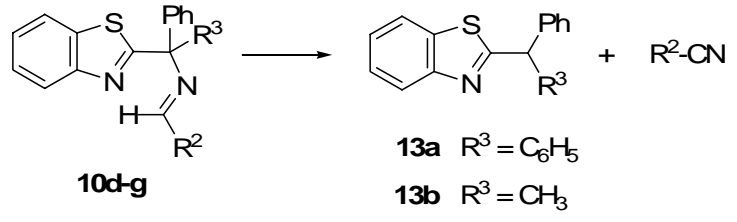

or by a conceptually similar fragmentation of the zwitterionic intermediates $\mathbf{1 1}$ shown in Scheme 5. Benzothiazoles 13 are known compounds. (13a): (a) Brembilla, A.; Roizard, D.; Lochin, P. Synth. Commun. 1990, 20, 3379. (b) Abayeh, O. J.; Olagbemiro, T. O.; Agho, M. O.; Amupitan, J. O. Bull. Soc. Chem. Belg. 1994, 103, 687. (c) Nutaitis, C. F.; ObazaNataitis, J. Org. Prep. Proced. Int. 1997, 29, 315. (13b): (a) Ramos, T.; Avendaño, C.; Elguero, J. J. Heterocycl. Chem. 1987, 24, 247. (b) Florio, S.; Troisi, L. Tetrahedron Lett. 1989, 30, 3721 .

18. CCDC 256791 contains the supplementary crystallographic data for 10a. The data can be obtained free of charge via www.ccdc.cam.ac.uk/conts/retrieving.html (or from the CCDC, 12 Union Road, Cambridge CB2 1EZ, UK; e-mail: deposit@ccdc.cam.ac.uk).

19. (a) Eguchi, S.; Matsushita, Y.; Yamashita, K. Org. Prep. Proc. Int. 1992, 24, 209. (b) Molina, P.; Vilaplana, M. J. Synthesis 1994, 1197.

20. For an example of Lewis acid catalyzed migration of an imino group from oxygen to carbon in O-arylketoximes, see: Kikugawa, Y.; Tsuji, C.; Miyazawa, E.; Sakamoto, T. Tetrahedron Lett. 2001, 42, 2337.

21. Bradshaw, T. D.; Wrigley, S.; Shi, D. F.; Schulz, R. J., Paull, K. D.; Stevens, M. F. G. Br. J. Cancer 1998, 77, 745.

22. For an excellent review on the synthesis of benzothiazoles, see: Ulrich, H. Product Class 18: Benzothiazoles and Related Compounds, Science of Synthesis 2002, 11, 835.

23. Benati, L.; Montevecchi, P. C. J. Org. Chem. 1981, 46, 4570.

24. Pickard, P. L.; Tolbert, T. L. J. Org. Chem. 1961, 26, 4886.

25. Taylor, E. C.; McKillop, A.; Hawks, G. H. Org. Synth. 1973, 52, 36.

26. Pracejus, H.; Wallura, G. J. Prakt. Chem. 1962, 19, 33. 